\title{
Novos registros de Orchidaceae para o Nordeste brasileiro: Acianthera tricarinata e Cyclopogon variegatus
}

\author{
Cecília Oliveira de Azevedo* \& Lucas Cardoso Marinho
}

Departamento de Ciências Naturais, Universidade Estadual do Sudoeste da Bahia, Estrada do Bem-Querer, Km 4, s/n, Bairro Universitário, 45.083-900, Vitória da Conquista, Bahia, Brasil.

\begin{abstract}
Resumo - Acianthera tricarinata e Cyclopogon variegatus, recentemente coletadas na Bahia, nos municípios de Barra do Choça e Vitória da Conquista, respectivamente, são aqui registradas pela primeira vez para o Nordeste do Brasil. Palavras-chave: Bahia, Barra do Choça, orquídeas, Vitória da Conquista.
\end{abstract}

\begin{abstract}
New records of Orchidaceae from the Northeast of Brazil: Acianthera tricarinata and Cyclopogon variegatus) - Acianthera tricarinata and Cyclopogon variegatus were recently collected in Bahia State, in the municipalities of Barra do Choça and Vitória da Conquista, respectively, extending their distribution to Northeast Brazil.

Additional key words: Bahia, Barra do Choça, orchids, Vitória da Conquista.
\end{abstract}

O Brasil possui uma das floras mais ricas do planeta. Estima-se que ocorram em seu território aproximadamente 56.000 espécies de plantas, cerca de $19 \%$ da flora mundial (Giulietti et al. 2005); porém, muitas áreas do país permanecem ainda inexploradas. Devido ao maior número de inventários florísticos realizados, o Sul e o Sudeste brasileiros possuem um conhecimento mais amplo de sua diversidade quando comparado a outras Regiões. A Região Nordeste, por exemplo, apesar de detentora de uma grande parcela do território nacional e de uma grande diversidade de ecossistemas, apresenta menos herbários e um menor número de espécimes coletados (Peixoto et al. 2006).

Os trabalhos que abordam a família Orchidaceae no Nordeste brasileiro (e.g., Harley \& Mayo 1980; van den Berg \& Azevedo 2005; Toscano de Brito \& Cribb 2005; Barros \& Felix 2006; Azevedo \& van den Berg 2007; Freitas et al. 2011; Barros et al. 2012) não citam a ocorrência de Acianthera tricarinata (Poepp. \& Endl.) Pridgeon \& M.W.Chase e Cyclopogon variegatus Barb.Rodr. O presente trabalho tem, então, o objetivo de indicar a ampliação da distribuição conhecida dessas espécies para o Nordeste do Brasil.

Foram consultados os principais herbários do estado da Bahia: ALCB, CEPEC, HRB, HUEFS e HUESB VC. O material coletado foi processado e depositado no herbário da Universidade Estadual do Sudoeste da Bahia (HUESBVC).

Acianthera tricarinata (Poepp. \& Endl.) Pridgeon \& M.W.Chase, Lindleyana 16(4): 246. 2001. Pleurothallis tricarinata Poepp. \& Endl., Nov. Gen. Sp. Pl. 1: 49; pl. 87. 1836.

= Pleurothallis platystachys Regel, Gartenflora 37: 459 . 1888. Acianthera platystachys (Regel) Chiron \& Van den Berg, Richardiana 12(2): 73. 2012.

Material examinado - BRASIL. BAHIA: Barra do Choça,

*Autora para correspondência: cazevedo@uesb.edu.br

Editor responsável: Alessandro Rapini

Submetido em: 16 ago. 2012; publicação eletrônica: 19 dez. 2012
Reserva do Hotel Fazenda São José, a ca. 12 km do município de Barra do Choça, 9 dez. 2011, C. Azevedo 406 (HUESBVC); ib., 9 jul. 2011, A.F.P. Machado \& A.K.A. Santos 1065 (HUEFS).

O gênero Acianthera está representado por 131 espécies (Chase et al. 2003), das quais 20 espécies ocorrem no Nordeste brasileiro (Barros et al. 2012). Acianthera tricarinata caracteriza-se por apresentar ramicaule, raque e flores lateralmente comprimidas, suas flores são esverdeadas com máculas vináceas (Figuras 1 e 2). O labelo é carnoso e trilobado, com calosidade formada por duas quilhas erguidas na porção mediana do lobo terminal (Figuras 1D e 2D/E). Morfologicamente, Acianthera platystachys é considerada sinônimo de A. tricarinata (Luer 2004; Barros et al. 2012). Em estudos recentes de filogenia molecular (Chiron \& van den Berg 2012; Chiron et al. 2012), as duas espécies apareceram em clados distintos, porém fracamente sustentados. Até que trabalhos com métodos específicos possam esclarecer essa questão com propriedade, estamos considerando $A$. platystachys sinônimo de A. tricarinata; Luer (2004) apresenta uma lista de sinônimos para essa espécie.

Acianthera tricarinata é conhecida para a Bolívia, Peru, Equador e Brasil (Luer 2004). No Brasil, foi citada para as Regiões Sul, com ocorrência no estado do Paraná, e Sudeste, onde é conhecida dos estados de São Paulo, Rio de Janeiro, Espírito Santo (Barros et al. 2012) e Minas Gerais (Menini-Neto et al. 2007); Pabst \& Dungs (1975) também citaram a espécie (como Pleurothallis platystachys) para as Regiões Sul e Sudeste. Acianthera tricarinata é aqui registrada pela primeira vez para o Nordeste, onde foi encontrada apenas no município de Barra do Choça, no estado da Bahia, em altitude aproximada de $1.100 \mathrm{~m}$. Recentemente, Acianthera saurocephala (Lodd.) Pridgeon \& M.W.Chase também foi registrada para a Região (Marinho \& Azevedo 2011).

Cyclopogon variegatus Barb.Rodr., Gen. Sp. Orchid. 2: 282. 1881. Spiranthes variegata (Barb.Rodr.) Cogn. in Martius

Sitientibus série Ciências Biológicas 12(2): 339-344. 2012. 

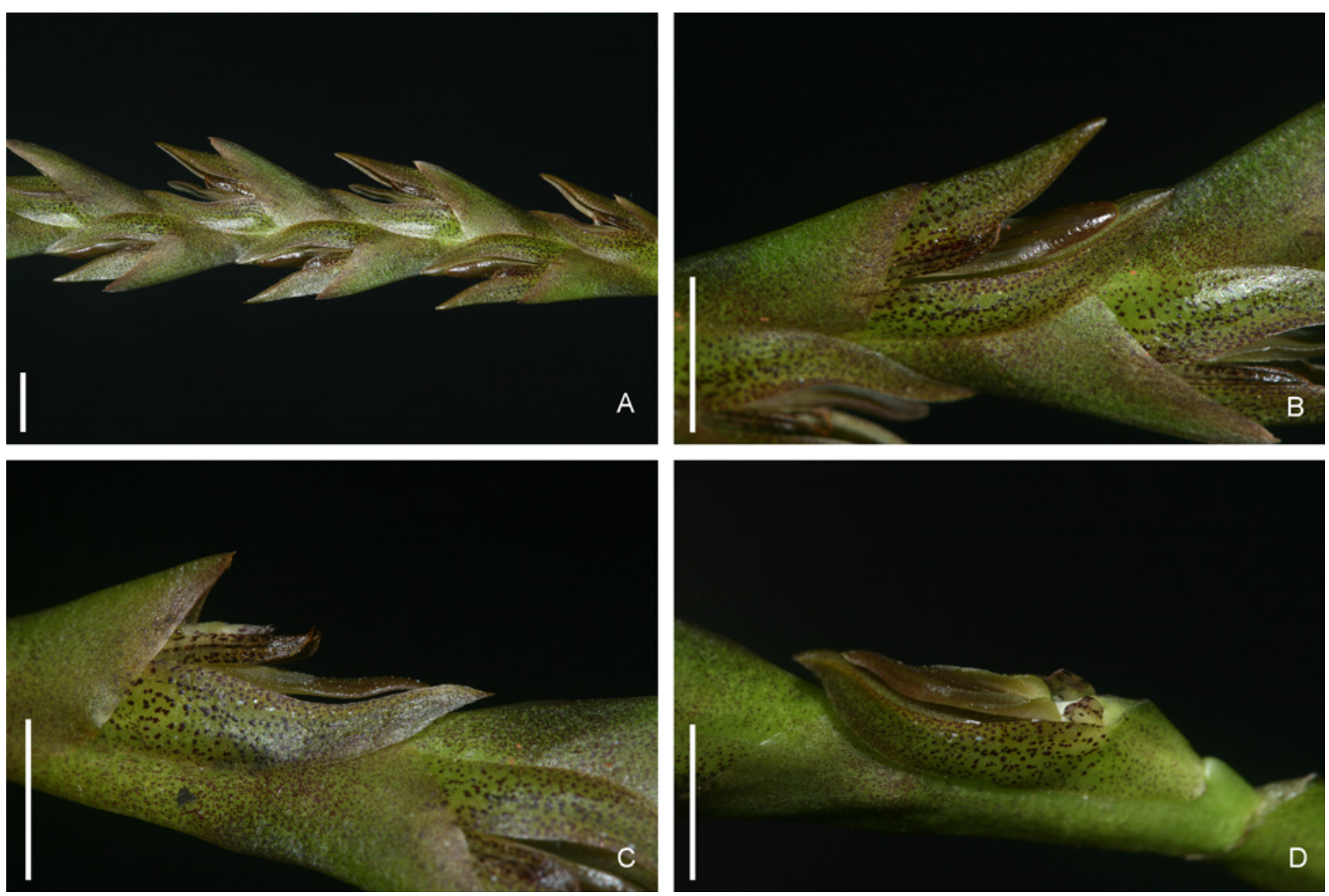

Figura 1. Acianthera tricarinata: A- inflorescência; B- flor; C- flor sem sépala dorsal; D- flor sem sépala dorsal, pétalas e coluna. Escala: 5 mm.

\& Eichler, Fl. Bras. 3(4): 193. 1895. Beadlea variegata (Barb.Rodr.) Garay, Bot. Mus. Leafl. 28(4): 301. 1982.

Material examinado - BRASIL. BAHIA: Vitória da Conquista, estância Terra das Rosas, a ca. $14 \mathrm{~km}$ da cidade, 18 ago. 2011, C. Azevedo et al. 380 (HUESBVC).

Cyclopogon possui 82 espécies (Chase et al. 2003), das quais 32 ocorrem no Brasil, e seis estão distribuídas no Nordeste brasileiro: Cyclopogon bicolor (Ker-Gaw.) Schltr., $C$. cearensis Barb.Rodr., C. congestus (Vell.) Hoehne, C. elatus (Sw.) Schltr., $C$. eldorado (Linden \& Rchb.f.) Schltr. e $C$. elegans Hoehne (Barros et al. 2012).

Cyclopogon variegatus é uma planta terrestre, com folhas dispostas em roseta, de coloração castanha com pontuações avermelhadas (Figuras 3A e 4A). A espécie é aparentemente rara, contudo o número reduzido de espécimes coletados pode estar relacionado à cor de suas folhas e ao tamanho reduzido de suas flores, uma vez que a planta confunde-se com a serrapilheira dificultando sua visualização (Figura 3A). Possui inflorescência pilosa, com flores dispostas em espiral. As brácteas florais são castanhas com pontuações brancas e as flores esverdeadas, apresentando pétalas de ápice branco com estrias avermelhadas e labelo branco, com lobo terminal levemente cuspidado (Figuras 3BD e 4). Segundo Hoehne (1942), a coloração das folhas, a inflorescência e o ovário pilosos, juntamente com as sépalas glabras, são características fundamentais para a identificação da espécie.

Cyclopogon variegatus distribui-se na Argentina e no Brasil (Pabst \& Dungs 1975; Johnson 2001), nas Regiões Sul, Sudeste e Centro-Oeste (Barros et al. 2012), presente nos estados do Rio Grande do Sul (Rocha \& Waechter 2006), Santa Catarina, Paraná, Rio de Janeiro e São Paulo (Pabst \& Dungs 1975), sendo aqui registrada pela primeira vez para o Nordeste. Encontrada geralmente em áreas de Floresta Ombrófila (Rocha \& Waechter 2006), foi coletada no sudoeste da Bahia, em área de Floresta Estacional Semidecidual. Entretanto, a região em questão apresenta maior gradiente de umidade que as áreas de semiárido ao seu redor, devido à maior incidência de massas úmidas no planalto de Vitória da Conquista (Queiroz et al. 2006).

\section{Agradecimentos}

A Anderson F. P. Machado, pela coleta do material botânico; a Antonio Toscano de Brito, pelas sugestões e comentários a este manuscrito; à Fundação de Amparo à Pesquisa do Estado da Bahia (FAPESB) e à Universidade Estadual do Sudoeste da Bahia (UESB), pelo apoio financeiro. 


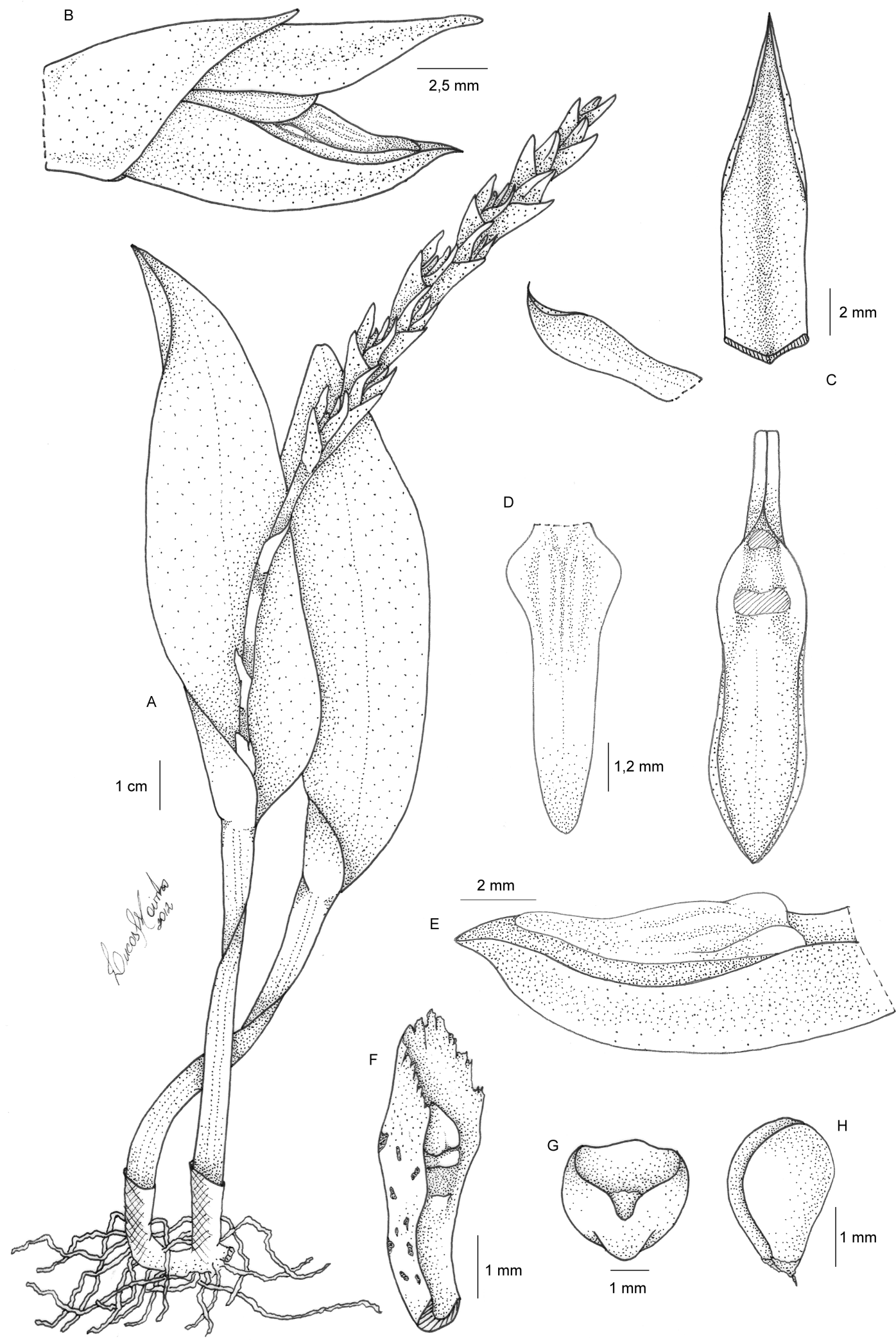

Figura 2. Acianthera tricarinata: A- hábito; B- flor; C- sépala e pétala; D- labelo estendido; E- sépalas laterais e labelo, vista lateral; F- coluna; Gantera; H- polinário. 

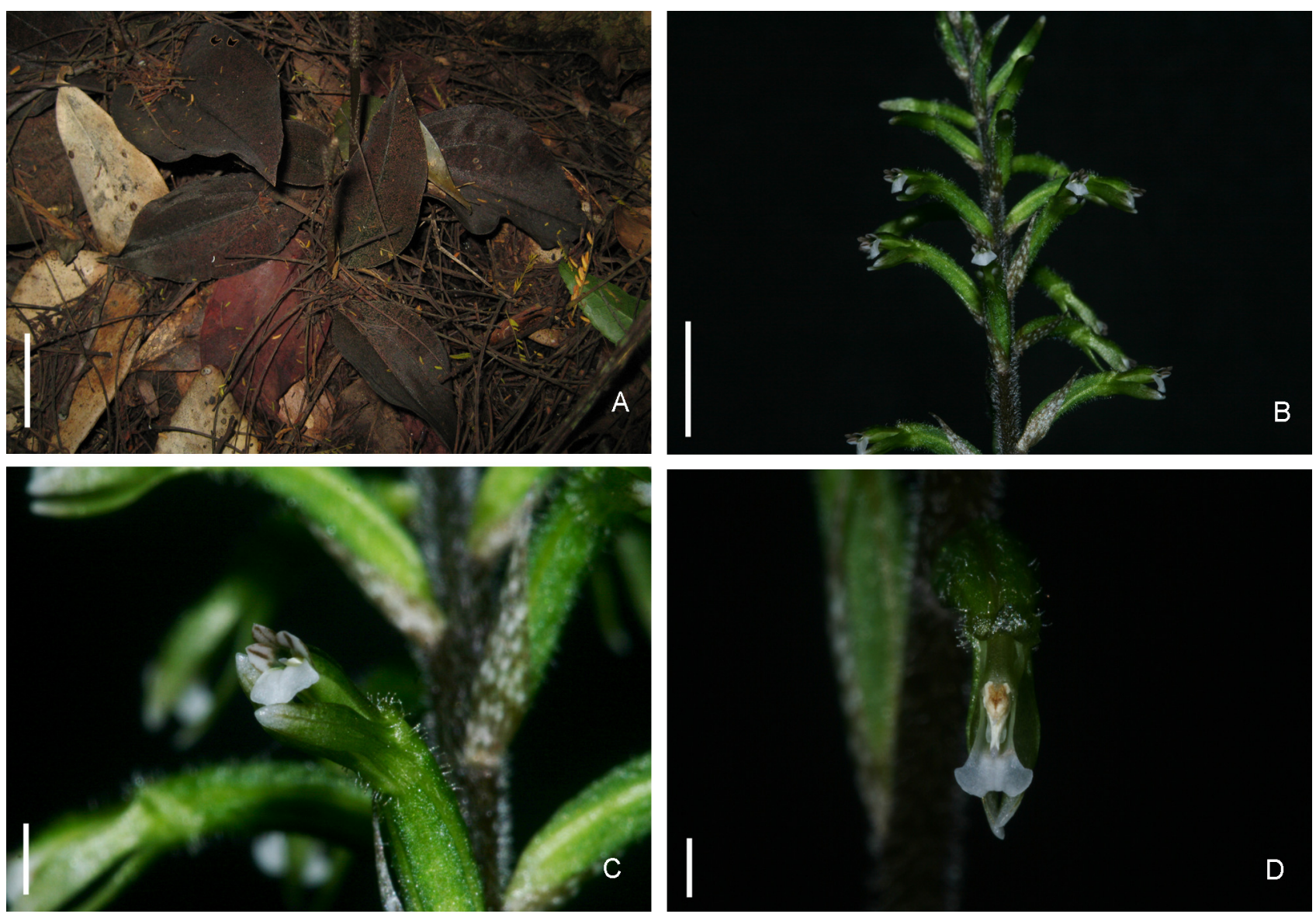

Figura 3. Cyclopogon variegatus: A- hábito; B- inflorescência; C- flor; D- flor sem sépala dorsal e pétalas. Escala: $\mathrm{A}=4 \mathrm{~cm}, \mathrm{~B}-\mathrm{D}=2 \mathrm{~mm}$.

\section{REFERÊNCIAS}

Azevedo, C.O. \& van den Berg, C. 2007. A família Orchidaceae no Parque Municipal de Mucugê, Bahia, Brasil. Hoehnea 34: $1-47$.

Barros, F. \& Felix, L.P. 2006. Orchidaceae. In: M.R.V. Barbosa, C. Sothers, S. Mayo, C.F.L. Gamarra-Rojas \& A.C. Mesquita (eds), Checklist das Plantas do Nordeste Brasileiro: angiospermas e gimnospermas. Ministério de Ciência e Tecnologia, Brasília, p. 82-89.

Barros, F.; Vinhos, F.; Rodrigues, V.T.; Barberena, F.F.V.A.; Fraga, C.N. \& Pessoa, E.M. 2012. Orchidaceae. In: Lista de Espécies da Flora do Brasil. Jardim Botânico do Rio de Janeiro. Disponível em http://floradobrasil.jbrj.gov.br/2012/ FB020183; acesso em 20 abr. 2012.

Chase, M.W.; Cameron, K.M.; Barrett, R.L. \& Freudenstein, J.V. 2003. DNA data and Orchidaceae systematics: a new phylogenetic classification. In: K.M. Dixon, S.P. Kell, R.L. Barrett \& P.J. Cribb (eds), Orchid Conservation. Natural History Publications, Sabah, p. 69-89.

Chiron, G.R. \& van den Berg, C. 2012. Révision taxinomique du genre Acianthera (Orchidaceae, Pleurothallidinae). Richardiana 12: 59-77.

Chiron, G.R.; Guiard, J. \& van den Berg, C. 2012. Phylogenetic relationships in Brazilian Pleurothallis sensu lato (Pleurothallidinae, Orchidaceae): evidence from nuclear ITS rDNA sequences. Phytotaxa 46: 34-58.
Freitas, R.C.A.; Santos, M.L.G. \& Matias, L.Q. 2011. Checklist das monocotiledôneas do Ceará, Brasil. Revista Caatinga 24: $75-84$.

Giulietti, A.M.; Harley, R.M.; Queiroz, L.P.; Wanderley, M.G.L. \& van den Berg, C. 2005. Biodiversidade e conservação das plantas no Brasil. Megadiversidade 1(1): 52-61.

Harley, R.M. \& Mayo, S.J. 1980. Towards a Checklist of the Flora of Bahia. Royal Botanic Gardens, Kew.

Hoehne, F.C. 1942. Orchidaceas. In: F.C. Hoehne (ed.), Flora Brasilica 12(6): 1-218.

Johnson, A.E. 2001. Las orquídeas del Parque Nacional Iguazú. Literature of Latin America (LOLA), Buenos Aires.

Luer, C.A. 2004. Systematics of Pleurothallis subgenus Acianthera (Orchidaceae). Icones Pleurothallidinarum XXVI Monographs in Systematic Botany from the Missouri Botanical Garden 95: $1-75$.

Marinho, L.C. \& Azevedo, C.O. 2011. Acianthera saurocephala (Lodd.) Pridgeon \& M.W.Chase (Orchidaceae: Pleurothallidinae), novo registro para o Nordeste brasileiro. Revista Brasileira de Biociências 9: 554-557.

Menini-Neto, L.; Alves, R.J.V.; Barros, F. \& Forzza, R.C. 2007. A subtribo Pleurothallidinae (Orchidaceae) no Parque Estadual de Ibitipoca, Minas Gerais, Brasil. Boletim de Botânica da Universidade de São Paulo 25: 253-278.

Pabst, G.F.J. \& Dungs, F. 1975. Orchidaceae Brasilienses. Vol. 


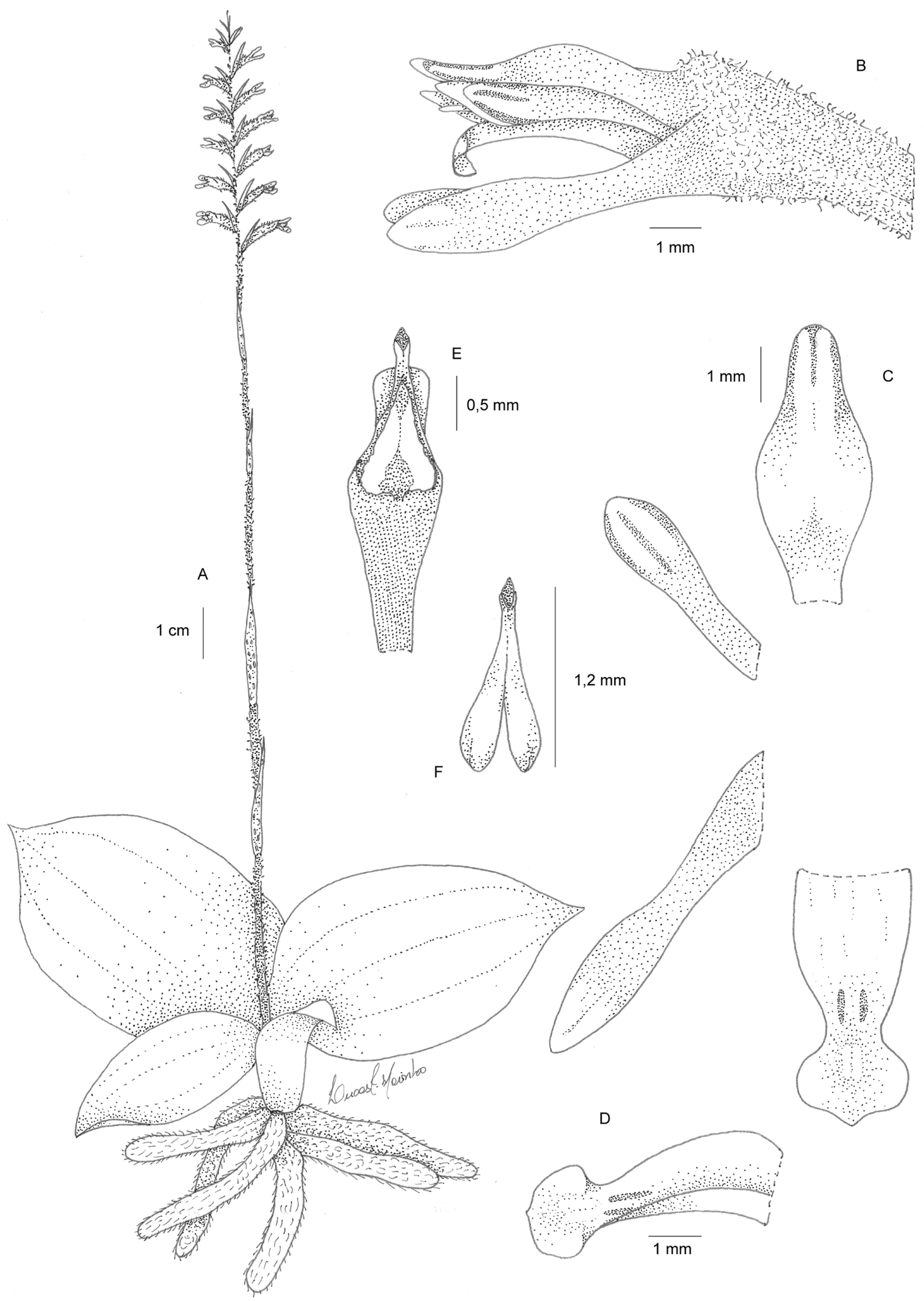

Figura 4. Cyclopogon variegatus: A- hábito; B- flor; C- sépalas, pétala e labelo estendido; D- labelo, vista lateral; E- coluna; F- polinário. 
1. Brücke-Verlag, Kurt Schmersow, Hildesheim.

Peixoto, A.L.; Barbosa, M.R.V.; Menezes, M. \& Maia, L.C. 2006. Diretrizes e estratégias para a modernização das coleções botânicas brasileiras com base na formação de taxonomistas e na consolidação de sistemas integrados de informação sobre biodiversidade. In: I. Egler, M.M. Santos \& V.P. Canhos (orgs), Diretrizes e Estratégias para a Modernização de Coleções Biológicas Brasileiras e a Consolidação de Sistemas Integrados de Informação sobre Biodiversidade. Ministério da Ciência e Tecnologia, Brasília, p. 145-182.

Queiroz, L.P.; Conceição, A.A. \& Giulietti, A.M. 2006. Nordeste Semi-árido: caracterização geral e lista das fanerógamas. In: A.M. Giulietti, A.A. Conceição \& L.P. Queiroz (orgs), Diver- sidade e Caracterização das Fanerógamas do Semi-árido Brasileiro. Vol. 1. Associação Plantas do Nordeste, Recife, p. 15-359.

Rocha, F.S. \& Waechter, J.L. 2006. Sinopse das Orchidaceae terrestres ocorrentes no litoral norte do Rio Grande do Sul, Brasil. Acta Botanica Brasilica 20: 71-86.

Toscano de Brito, A.L.V. \& Cribb, P. 2005. Orquídeas da Chapada Diamantina. Nova Fronteira, São Paulo.

Van den Berg, C. \& Azevedo, C.O. 2005. Orquídeas. In: F.A. Junca, L. Funch \& W. Rocha (eds), Biodiversidade e Conservação da Chapada Diamantina. Ministério do Meio Ambiente, Brasília, p. 195-208. 